\title{
The human gut virome database
}

2 Ann C. Gregory ${ }^{1, *}$, Olivier Zablocki ${ }^{1, *}$, Allison Howell ${ }^{1}$, Benjamin Bolduc ${ }^{1}$ \& Matthew B. Sullivan ${ }^{1,2, \#}$

$4 \quad{ }^{1}$ Department of Microbiology, Ohio State University, Columbus, OH, United States

$5 \quad{ }^{2}$ Department of Civil, Environmental and Geodetic Engineering, Ohio State University, Columbus, OH,

$6 \quad$ United States

$7 \quad{ }^{\#}$ Correspondence to: Matthew Sullivan, sullivan.948@ osu.edu

$8 *$ These authors contributed equally

9

10

11

\section{ABSTRACT}

The gut microbiome profoundly impacts human health and disease, but viruses that infect these microbes are likely also important. Problematically, viral sequences are often missed due to insufficient reference viral genomes. Here we (i) built a human gut virome database, GVD, from 648 viral particle metagenomes or microbial metagenomes from 572 individuals previously searched for viruses, (ii) assessed its effectiveness, and (iii) conducted meta-analyses. GVD contains 13,203 unique viral populations (approximately species-level taxa) organized into 702 novel genera, which roughly doubles known phage genera and improves viral detection rates over NCBI viral RefSeq nearly 60-fold. Applying GVD, we assessed and rejected the idea of a 'core' gut virome in healthy individuals, and found through meta-analyses that technical artifacts are more impactful than any 'treatment' effect across the entire meta-study dataset. Together, this foundational resource and these findings will help human microbiome researchers better identify viral roles in health and disease.

\section{Main text}

The human gut microbiome is now thought to play an integral role in health and disease ${ }^{1-4}$. Persistent alterations in the structure, diversity and function of gut microbial communities—dysbiosisare increasingly recognized as key contributors in the establishment and maintenance of a growing number of disease states ${ }^{5-7}$, including obesity ${ }^{8}$ and cancer ${ }^{9}$. Gut dysbiosis can develop from complex 
27 interplays between host, cognate microbiota and external environmental factors ${ }^{10,11}$. Within the gut

28 microbial consortium, the bacteriome has been the most extensively studied, where significant shifts in

29 population dynamics have been observed between healthy and diseased individuals ${ }^{12}$. However,

30 emerging views ${ }^{10,13,14}$ suggest that the gut virome plays an important role in homeostatic regulation and

31 disease progression through multiple interaction paths with the co-occurring bacteriome, and even

32 directly with human immune system components ${ }^{15}$.

33 The first step in studying viruses in complex communities is to "see" them. Problematically,

34 identifying viral sequences in large datasets is notoriously challenging. Because viruses lack a universal

35 viral marker ${ }^{16}$, as opposed to bacterial 16S rRNA for example, researchers often resort to sequence

36 homology searches against reference databases (e.g. NCBI viral RefSeq). Such searches are variably

37 successful with anywhere from $14 \%$ to $87 \%$ of the observed gut viral genomes having detectable

38 similarity to viruses in such databases ${ }^{10}$. This large range stems from several factors that are not

39 mutually-exclusive including the following: (i) broad under-representation of viral genome space in

40 databases, (ii) non-standardized database usage per study, (iii) overrepresentation of certain virus groups

41 due to sample preparation and cultured host availability, and (iv) natural sample variation. In addition,

42 although viral reference datasets are being generated at unprecedented rates ${ }^{17}$, these new data are rarely

43 incorporated for cross-comparisons, which inflates virus novelty in new datasets and/or leaves many virus

44 sequences undetected. Therefore, given the rapid accrual of so many studies, there is a need to aggregate

45 their findings into a central gut-specific database to improve gut virome inference capabilities.

Here we collected and curated 648 gut metagenomes from 21 datasets (i.e., any metagenomic

47 dataset that looked at gut viruses published before 2018), consistently processed them to map known and

48 unknown viral populations, and used this in multiple meta-analyses to assess improvement and reveal

49 new biology. The resulting Gut Virome Database (GVD) was born by (i) collecting 648 gut metagenomes

50 from 572 individuals, (ii) extensive metadata curation through literature mining and, as needed, direct

51 communication with the original researchers, and (iii) re-analysis of the virome data to establish

52 consistent processing and extensive virus identification. The value of GVD was assessed for performance 
53 against the best currently available databases (NCBI viral RefSeq and IMG/VR ${ }^{18}$ ), and then used to re-

54 evaluate global diversity patterns and the relationship between gut virome diversity and diet.

56 RESULTS AND DISCUSSION

GVD contains 13,204 viral populations, dominated by phages

59 To build a collection of the commensal human gut virome, 648 metagenomic samples from 572

60 individuals were processed from all datasets publicly available as of December $2017(\mathrm{n}=19)$, along with 2

61 unpublished datasets where access was granted prior to publication. These studies represented a total of

$621.28 \mathrm{Tbp}$ of sequence data derived from a spectrum of gut virome study areas including: (i) healthy gut

63 viromes of infants ${ }^{19,20}$ and adults ${ }^{21-26}$, as well as individuals experiencing (ii) fecal matter transplant, or

64 FMT $^{27-31}$, (iii) inflammatory bowel disease, or IBD ${ }^{32,33}$, (iv) HIV infection ${ }^{34}$, (v) Type I diabetes ${ }^{35,36}$,

65 (vi) malnutrition ${ }^{37}$, or (vii) chronic fatigue syndrome ${ }^{38}$ (see Supplementary Table 1). Datasets had a

66 worldwide distribution, though most originated from the United States (48.4\%; Fig. 1a). All reads were

67 processed consistently, assembled into contigs and viral-like sequence were identified using three

68 independent methods and validated by cross-comparisons between methods (Fig. 1b, see Methods). To

69 avoid duplicate viral fragments/partial virus genomes across the datasets, contigs were de-replicated by

70 clustering sequences according to percentage of average nucleotide identity (ANI) and sequence length.

71 Multiple reports ${ }^{17,39-43}$ have revealed that $>95 \%$ ANI was a suitable threshold for defining a set of

72 closely-related discrete 'viral populations', with follow-on studies suggesting that this cut-off establishes

73 populations that are largely concordant with a biologically relevant viral species definition ${ }^{39,41,44}$. Using

74 this clustering strategy, we identified highly variable numbers of unique viral populations per study

75 (range: 0 - 3596; mean = 670) $($ Supplementary Fig. 1a). GVD comprises 13,203 viral populations (N50

$76=34,220$ bp ; L50 = 2,066 bp). For context, NCBI's viral RefSeq v88 (released May 2018) database holds

778,013 viruses of eukaryotes, bacteria and archaea from all environments, combined. Moreover, if only

78 comparing phage genomes to the same database, GVD contains 7 times more phages compared to the 
entire set of cultured phage isolates in viral RefSeq to date. Thus, GVD greatly augments the repertoire of known viruses in the human gut. minority of GVD viral populations more likely to represent eukaryotic viruses (3.8\%) and archaeal viruses $(0.1 \%)$ (Fig. 2a). Though in the minority, the 505 eukaryotic viruses were taxonomically diverse

84 (14 families), dominated by ssDNA families Anelloviridae (72\%), Genomoviridae (10\%) and

85 Circoviridae (8\%). All, with the exception of Genomoviruses, have been reported previously in the 86 datasets underlying GVD ${ }^{34}$. Among the phages, $82 \%$ did not have ICTV classification, with the

87 remaining fraction comprised of dsDNA tailed phage families (Siphoviridae, Myoviridae and

88 Podoviridae), Microviridae and Inoviridae (see Supplementary Table 2). Twelve unknown archaeal

89 viral populations were detected, with no close genome/gene homology to any of the classified archaeal

90 viruses. The high number of unclassified phages likely results from underrepresentation of gut phages in

91 the database, coupled to unresolved and/or missing taxonomic assignments for $\sim 60 \%$ of reference phage

92 genomes in RefSeq, with the currently classified fraction organized into $\sim 250$ genera ${ }^{45}$. To fill this phage

93 and archaeal virus taxonomic classification gap, we used a genome-based, gene-sharing network strategy

$94{ }^{46,47}$ that de novo predicts genus-level groupings ('viral clusters' or 'VCs') from viral population data. A

95 network was computed from 6,373 GVD phage genomes (only those $\geq 10 \mathrm{~kb}$ in length; $48 \%$ of GVD),

96 combined with 2,304 curated reference phage genomes from NCBI Viral RefSeq (version 88). The

97 resulting gene-sharing network (Fig. 2b) revealed 957 VCs, 702 of which were novel and exclusively

98 composed of GVD genomes (3,220 viral genomes or $~ 51 \%$ of GVD genomes). This would roughly

99 double the current number of ICTV-recognized phage genera. Though not explored here, as our goals

100 focused on taxonomic classification, the shared protein content within and between VCs calculated in our 101 network analyses could be used to guide qPCR assays for NGS validation ${ }^{48}$ and/or tracking of viruses at 102 either the viral population- or genera- level under changing conditions ${ }^{35}$.

103 Next, we sought to link phage populations to their hosts using in silico strategies (see Methods).

104 The most common identifiable phage hosts (Fig. 2c) in GVD belonged the bacterial phylum Firmicutes 
105 (38\%), about 2-fold more than the next most abundantly identified host phyla (Bacteroidetes and

106 Proteobacteria; see Supplementary Table 2). Though Firmicutes and Bacteroides are the most prominent

107 bacterial phyla in the human gastrointestinal tract ${ }^{49}$, Firmicutes typically outnumber Bacteroidetes in

108 unhealthy individuals with metabolic and digestive disorders ${ }^{50-52}$. GVD metagenomes originated from

$109 \sim 16 \%$ healthy individuals and $~ 84 \%$ unhealthy individuals, many of which have metabolic and digestive

110 disorders. Thus, it is perhaps not surprising that most of the annotated viral populations were linked to the

111 phylum Firmicutes.

113 GVD significantly improves virus detection in all gut datasets

114 We then quantitatively evaluated virus identification sensitivity (through read mapping) between

115 multiple databases by comparing the number of identified viral populations in each study detected by

116 GVD, viral RefSeq v88, IMG/VR 1.1 (2018 release) and the individual virome datasets ('IV') from each

117 study (Fig. 3). For the latter, IV reads were mapped against viral populations (predicted in this study)

118 derived exclusively from its matching IV. In all datasets, GVD surpassed viral RefSeq (mean increase:

119 59-fold \pm 95-fold) and IVs (mean increase: 3.2 -fold \pm 6.6 -fold). In 5 of 18 studies (28\%), GVD

120 outperformed IMG/VR (mean increase: 1.1 -fold \pm 2 -fold), with the remaining studies finding no

121 significant difference between or too low of a sample size to compare GVD and IMG/VR. After GVD,

122 IMG/VR was the next best performing database for viral detection in the gut, as our tests showed an

123 average of 49-fold ( \pm 87-fold) increase over viral RefSeq. IMG/VR was expected to surpass viral RefSeq,

124 as it aggregates both cultivated reference virus genomes, $>12,000$ prophages and $>700,000$ uncultivated

125 virus genomes/fragments from many environments, including multiple human body sites ${ }^{53}$. Moreover,

126 given the high performance of IMG/VR in our tests, we wondered about the extent of viral population

127 overlap with GVD (Fig. 3b). There were 1,730 viral populations shared between the two databases, but

128 still each database is overwhelmingly unique (82\% and 69\% unique to GVD and IMG/VR, respectively).

129 This is because IMG/VR includes human gut studies that did not explore the viral fraction as well.

130 Overall, the significant increase in virus detection by GVD over other databases (two-tailed Mann- 
131 Whitney U-tests; $p$-value < 0.05) highlights the low representation of gut viruses recorded in RefSeq and

132 thus demonstrates the value of GVD for sequence-based virus identification in human gut microbiome

133 datasets. Because the datasets used to compile GVD were originally analyzed most often (55\% of the

134 studies) using viral RefSeq as the primary source to identify viruses (Supplementary Table 1), we

135 wondered whether significant fractions of viruses could have been missed, and whether a possibly

136 reduced viral "signal" would influence previous conclusions.

MDA amplification skews diversity and prohibits quantitative analysis of gut viromes

To evaluate this possible reduced viral "signal", we first examined the role of methodological

140 approaches in influencing inferences about ssDNA viruses. This is because we noticed that the bulk of

141 ssDNA eukaryotic viruses (Anelloviruses, Circoviruses, Genomoviruses, Geminiviruses) and phages

142 (Microviruses) originated from only 4 of the 21 studies gathered in this work (Fig. 4 a,b). These studies

143 evaluated 2 infant gut viromes ${ }^{19,37}$ and 2 adult inflammatory bowel disease viromes ${ }^{31,32}$, and they

144 reported relative abundance shifts of ssDNA and dsDNA phages within these viromes. From this

145 observation, these studies concluded that such shifts could discriminate between healthy and disease

146 states associated with virome development in early life.

147 However, the abundance of ssDNA viruses can also be enriched from methodologies used in

148 making the viromes, even if all samples are processed consistently. Specifically, early virome studies

149 where limiting viral nucleic acids were obtained, often used whole genome amplification kits that

150 leverage a DNA polymerase from the phi29 ssDNA virus to obtain many-fold increases in DNA via

151 multiple displacement amplification or MDA ${ }^{54}$. Though attractive at first, MDA is now known to have

152 stochastic biases (e.g., 100s $-10,000$ s-fold biases in coverage, ${ }^{55,56}$ ), which result from randomized initial

153 template interactions and can induce chimera formation and uneven amplification of linear genomic

154 sections (whether ssDNA or dsDNA templates), as well as systematic biases resulting from preferential

155 amplification of small, circular and ssDNA genomes ${ }^{57-61}$. Taken together, MDA-associated artifacts skew

156 the taxonomic representation of a community in non-repeatable ways and preclude quantitative analysis 
157 of viromes ${ }^{57}$. Although non-quantitative, MDA-amplified viromes do still have value enriching for

158 ssDNA viruses, as well as estimating presence of viruses.

Consistent with the idea that these ssDNA viruses are methodologically enriched in the MDA

160 libraries, we found that non-MDA amplified gut viromes contained significantly less ssDNA viruses than

161 MDA amplified gut viromes (range: $0 \%$ - 4\% versus 0-42\%; Mann-Whitney U-test; $p$-value $=0.0083$ ),

162 though sample size was quite low. Further, while we see a strong linear relationship $\left(\mathrm{R}^{2}=0.86\right)$ between

163 sequencing depth and the number of viral populations sequenced in non-MDA viromes, this relationship

164 is weak in MDA viromes $\left(\mathrm{R}^{2}=0.39\right)$, suggesting that MDA can skew the number of assembled viral

165 contigs in datasets (Supplementary Fig. 1b). Critically, 14 of the 21 studies gathered in this work

166 employed MDA, which calls into question the quantitative nature of these datasets. Fortunately, viral

167 nucleic acid extraction from feces often yield sufficient quantities for high throughput sequencing ${ }^{26}$, and

168 in cases where they do not there are now several viable alternative methods to more quantitatively

169 establish viromes with as little as $1 \mathrm{pg}$ of DNA ${ }^{61,62}$. Problematically, current established gut virome

170 protocols recommend an MDA step ${ }^{48,63}$. If a researcher's goal is to provide quantitative datasets, then we

171 strongly advocate against this recommendation and instead suggest that alternative methods ${ }^{61,62}$ be used to

172 generate gut viromes.

174 Human gut virome study conclusions are more impacted by methodology than disease state

175 Given a systematically processed GVD, we next sought to determine whether global clustering patterns

176 would emerge between study themes between all dataset used to build GVD. To this end, viral

177 populations identified in this study were matched back to their respective datasets, and used in a co-

178 occurrence network analysis (see Methods) to assess co-variation at two levels: between study datasets

179 (Fig. 4c), and between viromes across all datasets (Fig. 4d). Between datasets, the fraction of shared viral

180 populations was low (mean: $3 \% \pm 3 \%$; Fig. 4c), except for 6 datasets that clustered together (hierarchical

181 clustering bootstrap $=100 \%$; Fig. 4 c) and had a higher level of shared viral populations (>4-fold

182 increase). Presumably, these elevated similarities across the 6 datasets may be due to deeper sequencing 
183 (Fig. 4d, top panel) that allowed deeper sequencing into the rare tail of viral populations among samples.

184 A similar trend was observed when looking at the level of individuals within each study (Fig. 4d), where

185 the co-occurrence network revealed close clustering between individuals derived from the same study,

186 irrespective of geographical origin, health status and/or diet. This per study clustering implies that, taken

187 together, these studies are not comparable likely due inconsistent sampling and extraction methodologies.

188 We then investigated the prevalence of gut viral populations amongst all samples, so as to establish

189 whether any viral populations were detected in all samples (i.e., a 'core' gut virome ${ }^{22}$ ). On average, 138 \pm

190170 (average \pm SD; range: 0 to 849 ) viral populations were detected per sample, but not one viral

191 population was found across all samples. We then explored deeper to detect whether subsets of the

192 samples would reveal shared viral populations. We found that only 28 viral populations occurred in over

$19320 \%$ of the GVD samples. Most viral populations were detected in very few samples. In fact, $>40 \%$ of

194 the viral populations occurred in $<0.5 \%$ of the samples and $98 \%$ of the viral populations occurred in

$195<0.1 \%$ of the samples in GVD (Fig. 5 a, b and Supplementary Table 3). Further, we specifically looked

196 at the prevalence of crAssphages, a well-recognized, multi-genera group of phages known to be

197 widespread in gut viromes ${ }^{64}$ (Fig.5 b, c). While crAssphages are ubiquitous across the GVD samples,

198 there was not one crAssphage viral population found universally, with the most widespread crAssphage

199 population occurring in only $38 \%$ of samples. Importantly, when we looked at all healthy samples and

200 healthy western samples specifically, still no shared viral populations were identified in all samples.

201 (Supplementary Fig. 2a, b). Assuming samples were sufficiently sequenced, this may be indicative that

202 individuals carry a unique 'gut virome fingerprint', even between twins, which is perhaps not surprising

203 given recent suggestions of a similar 'fingerprint' for gut microbes (the 'personal' microbial microbiomes

$204^{65}$ ). This apparent lack of core gut virome among individuals contrasts with a recent report ${ }^{22}$, in which

205 overlapping patterns of phage genomes between 2 unrelated healthy individuals, as well as within a re-

206 analyzed larger cohort ${ }^{66}$ revealed three levels of sharing patterns: (i) core (phage found in $>50 \%$ of

207 samples, (ii) common (phage found in $>20-50 \%$ of samples), and (iii) unique (phage found in $<20 \%$ of

208 samples). Our analyses showed no viral populations shared above $>50 \%$ of samples, thus bringing into 
209 question the presence of a 'core' virome as previously defined ${ }^{22}$, as well as a very limited 'common'

210 virome (20-50\% sharing across samples), in which we observed either $1 \%$ (all healthy; $\mathrm{n}=132$ ) or $0.1 \%$

211 (all healthy Westerners; $n=18$ ) of GVD viral populations, similar to the $3 \%$ previously reported ${ }^{22}$ (see

212 Supplementary Table 4). Likely, this discrepancy with our results could be attributed to how viruses

213 were identified through read mapping. In the initial study reporting a core virome ${ }^{22}$, a virus was

214 considered present if a single read mapped to a genome, a very permissive cut-off which does not take

215 into account shared homologous regions between distinct viral populations. In this study, we considered

216 a virus present if reads mapped $70 \%$ of the genome length (if genome is $<5 \mathrm{~kb}$ ) or reads mapped at least

$2175 \mathrm{~kb}$ of the genome (for genome $>5 \mathrm{~kb}$ in length) (see Methods). While our cut-off is more conservative, it

218 better ensures that we are detecting the same viral population. Nonetheless, the idea of a core virome

219 might still be an open question.

Re-evaluation of a previous study: the virome across different geographic regions and lifestyles

Due to the high level of sample clustering per study (Fig. 4c), we were unable to conduct cross-

Fig. 6a), and explored the impact of geography and diet on eukaryotic gut viruses (but did not include

229 phages) and found that the hunter-gatherers (Hadza in Tanzania and the Matses in Peru) had the highest

230 eukaryotic viral richness ${ }^{26}$.

In this re-analysis, however, we included phages in addition to eukaryotic viruses, and focused on

232 how the virome diversity varied along the dataset. We first evaluated whether per-region GVD-mediated

233 detection of viruses would incur biases, potentially stemming from underrepresented viral populations

234 from less-sampled geographical regions. This did not appear to be the case, as significant increases in 
235 virus detection were observed across 4 out of the 5 regions sampled (Fig. 6b). We next calculated

236 diversity indices (Fig. 6c and Supplementary Fig. 3) for each regional dataset, and looked at the number

237 of viral populations mapped with GVD. Overall, we reached a similar conclusion to the initial study (even

238 when considering phages), in which the hunter-gatherers (Peru Matses) generally contained higher viral

239 richness (Fig. 6c - left) and biodiversity (Shannon's H, Fig. 6c - middle), but not higher evenness

240 (Peilou's J, Fig. 6c -right). Collector's curves revealed that we have not saturated the human gut viral

241 diversity among individuals globally (Supplementary Fig. 4) or even among just among American

242 samples (Supplementary Fig. 2, inset). Thus, it appears much more viral diversity remains to be

243 discovered across all geographic regions.

We next wondered whether the addition of phage in our analysis would reflect on overall viral

245 community similarities by using Bray-Curtis distances between individuals across these geographic and

246 lifestyle gradients (Fig. 6d). While unequal database representation can have an impact on alpha-

247 diversity, beta-diversity is often less impacted ${ }^{67}$. Principal coordinate analyses (PCoA) of Bray-Curtis

248 distances derived from using the individual Rampelli et al., 2017 virome database (Fig. 6d, left panel) and

249 GVD (Fig. 6d, right panel) revealed no significant differences (Mantel's test; $\mathrm{R}=0.95, p=0.001$ ).

250 However, analysis of the GVD-referenced PCoA revealed individuals with the same lifestyle and from

251 the same region clustered together (PERMANOVA; $p \leq \square 0.001$ ) and provided better resolution of the

252 clustering in comparison to the IV-referenced PCoA. However, lifestyle alone may not account for the

253 observed clustering patterns. The viromes of the Hadza in Tanzania and semi-industrialized, agrarian

254 Tunapuco population in Peru strongly overlapped (hierarchical clustering bootstrap = 100\%; Fig. 6d),

255 most likely driven by their diets rich in root vegetables ${ }^{68-70}$. Nonetheless, when we look at differences

256 between dominant viral populations (found in $>50 \%$ individuals) across these geographic and lifestyle

257 gradients, we see that there are key viruses missing from Western, industrialized gut viromes (Fig. 6e),

258 specifically viruses that infect the genus Prevotella spp. This parallels the bacterial analyses that show

259 that Prevotella spp. are enriched in non-Western gut microbiomes and many species are missing from 
260 Western, industrial gut microbiomes ${ }^{69-71}$. Overall, this suggests that lifestyle and diet has an impact not

261 only on the bacterial community, but also on the viral community in the gut.

\section{CONCLUSIONS}

The lack of a curated database for the detection of viral sequences in the human gut has been

264 identified as the most critical shortcoming of applying metagenomic approaches to studying the human

265 gut virome ${ }^{72}$. Although GVD is geared towards filling this gap and performs well (increasing viral

266 detection 59-fold over the most commonly used database, NCBI viral RefSeq), there are limitations. First,

267 the geographic and ethnic representation across the dataset is not very broad. Meta-analyses will benefit

268 from more broadly representative datasets. Second, GVD was built using all datasets available by the end

269 of 2017. Since then, as of May 2019, there are 11 additional datasets that study the gut virome, 8 of which

270 use viral particle-enriched metagenomes (Supplementary Table 5). Further, there are many more human

271 gut microbial metagenomic datasets and these could be a rich source for virus reference genomes as found

272 for soils ${ }^{73}$ and the large-scale Earth Virome study ${ }^{74}$. To maintain significance as a resource, we will

273 update GVD annually by extracting the viral signal from such gut-related datasets, as well as monitoring

274 IMG/VR for gut-related viruses that should be integrated. Third, GVD is accessible through direct

275 download as a single fasta file containing all GVD viral populations (see link in the 'Data availability'

276 statement below), and is likely best paired with IMG/VR to maximize viral signal recovery. Future GVD

277 updates and development will be required to improve the user experience for those not comfortable at

278 command-line interfaces, but these are likely best integrated with large-scale standardizing efforts like the

279 National Microbiome Initiative.

Given the relatively minimal value added via non-quantitative MDA-based approaches and the

281 availability now of low-input quantitative approaches pioneered studying ocean viruses ${ }^{61,75}$ suggest that

282 gut virome studies should move away from the former towards the latter. GVD, combined with the means

283 to classify uncultivated virus genomes ${ }^{47}$, are prime starting requirements for enabling ecosystem-wide

284 examinations $^{76}$ of the dynamics and impacts of the virome within the human gut. Other environmental

285 advances also invite such studies to include assessing the role of micro- and macro-diversity on virus 
286 persistance ${ }^{41}$, and metabolic reprogramming via virus-encoded auxiliary metabolic genes ${ }^{73,76}$. These

287 combined efforts are critical to enable studies of the human gut virome to advance from 'stamp

288 collecting' diversity studies towards the kinds of comprehensive efforts needed to incorporate viruses into

289 mechanistic, predictive models. Such efforts, with future viral mapping outside the gut to parallel efforts

290 for the 'non-gut' human microbiome ${ }^{77}$, should help transform personalized medicine and lead to a better

291 understanding of human ecosystems.

292 
Figure 1. Overview of studies and meta-analyses comprising the Gut Viral Database (GVD). (a) Global heatmap of the world showing the number of individual's gut viromes coming from different countries within the GVD. Importantly, individual's viromes coming from the Cameroon were pooled based on their location, age, and contact with bats. The pools were counted as a single individual's virome for our analyses. (b) Pipeline for the selection and processing of human gut virome datasets (see Methods). Datasets were processed individually and, within each dataset, viromes were pooled by individual, except for fecal microbiota transfer (FMT) studies and data that was given to us prior to publication (Yinda et al., 2019; Neto et al. (unpublished)). Reads were filtered for quality and trimmed and reads that mapped to $\Phi x 174$ and the human genome were removed. The remaining reads were assembled into scaffolds, filtered for lengths $\geq 1.5 \mathrm{~kb}$, and run through tools that collectively utilize homology to viral reference databases, probabilistic models on viral genomic features, and viral $k$-mer signatures to identify viral contigs. Viral contigs were then deduplicated to get a total of 13,203 viral populations.

Figure 2. The Gut Viral Database (GVD). (a) Pie charts showing the number of bacteriophages, eukaryotic viruses, and archaeal viruses in the GVD (center) and their familial taxonomic composition by the bacteriophages (left) and the eukaryotic viruses (right). (b) Gene-sharing taxonomic network of the GVD, including viral RefSeq viruses v88. RefSeq viruses are highlighted in red. Every node represent a virus genome, while connecting edges identify significant gene-sharing between genomes, which form the basis for their clustering in genus-level taxonomy. (c) Bar chart showing the number of bacterial host phyla of the GVD bacteriophages, with an inset providing resolution for the low frequency bacteria host phyla. Putative host phyla per each bacteriophage population are in Supplementary Table 3

Figure 3. GVD as a reference database increases viral population detection. (a) Boxplots showing median and quartiles of the number of viral populations detected per study using the IV, Viral Refseq v88, JGI IMG/VR, or GVD databases. Studies where the reads were given to us prior to publication are excluded from this analysis (Yinda et al., 2019; Neto et al. (unpublished)). (b) Venn diagram showing the number of viral populations unique and shared between the different databases. Importantly, we only compared dereplicated viral populations from IMG/VR that came directly from human gut samples or had reads mapping to them from GVD gut samples.

Figure 4. Individual Viromes (IV) Study Databases and Cross-Study Comparisons. (a) Barplot showing the proportion of those viruses that are bacteriophages, archaeal viruses, or eukaryotic viruses. The total number of assembled viral contigs and viral populations per study are available in Supplementary Fig. 1a. (b) Barplot showing the proportion of those viruses that are dsDNA, ssDNA, or RNA viruses. Studies where multiple displacement amplification (MDA) was used show a higher prevalence of ssDNA viruses. No viral contigs $\geq 1.5 \mathrm{~kb}$ were assembled from the Reyes et al. 2010 study. (c) Hierarchically clustered heatmap showing the number of viral populations shared within and between studies. The barplot on top of the heatmap shows the total number of sequenced base pairs following quality control within each study. (d) Viral population co-occurrence network per individual within each study shows that individuals within a study cluster together regardless of health status. The squares represent the healthy individuals within each study.

Figure 5. There are no core viral populations across GVD samples. (a) Histogram showing the number of viral populations present in different percentages of GVD samples. The vast majority of viral populations are found in $<10 \%$ of the individuals. (b) Hive plot showing the percentage of GVD sample each viral population is detected within. The dots on the $\mathrm{x}$-axis represent each GVD viral population in ascending order of the percentage of GVD samples that they are found within. The y-axis is the percentage of GVD samples that each viral population is detected within. CrAssphage viral populations 
are highlighted in red. (c) Heatmap showing the presence or absence of each crAssphage viral population across the different GVD samples.

Figure 6. Diet and geography widely influence gut virome. (a) World map showing the geographical distribution of the Rampelli et al., 2017 dataset. (b) Boxplots showing median and quartiles of the number of viral populations detected using the GVD database and the Rampelli et al., 2017 viral database alone (IV) within each geographic group. (c) Boxplots showing median and quartiles of the $\alpha$-diversity metrics - richness, Shannon's H and Peilou's J - across the different geographic groups using the GVD database (see Fig. S3 for $\alpha$-diversity metrics using the IV database). (d) Principal coordinate analysis (PCoA) of a Bray-Curtis dissimilarity matrix calculated from mapping the Rampelli et al., 2017 dataset against the IV (left) and GVD (right) databases. Analyses show that the viromes significantly (Permanova $\mathrm{p}<0.05$ ) structure into based on the geographic groups, with mapping to the GVD showing revealing much stronger clustering based on geography. Ellipses in the PCoA plot are drawn around the centroids of each group at a $95 \%$ confidence interval. The dashed lines connecting the different points reveal the connections determined by hierarchically clustering between the different samples. (e) Heatmap of the abundances of the viral populations found across $>50 \%$ of individuals within the study. Individuals on a Western diet (from the USA and Italy) lack phages that infect Bacteroidetes, specifically those that infect Prevotella sp. All pairwise comparisons were performed using a two-tailed Mann-Whitney U-tests.

Supplementary Figure 1. Number of assembled viral contigs and populations. (a) Barplot showing the number of assembled viral contigs versus the number of deduplicated viral populations per study. (b) Scatterplots with linear regressions showing the impact of increased sequencing on the number of assembled contigs per study divided by studies that did not have multiple displacement amplification (MDA; top) and those that did have MDA (bottom).

Supplementary Figure 2. There are no core viral populations across healthy samples and across healthy western samples. Hive plots showing the percentage of GVD samples each viral population is detected within across (A) all healthy individuals and (B) across only healthy western adults. The dots on the $\mathrm{x}$-axis represent each GVD viral population in ascending order of the percentage of GVD samples that they are found within. The y-axis is the percentage of GVD samples that each viral population is detected within.

Supplementary Figure 3. Boxplots showing median and quartiles of the $\alpha$-diversity metrics - richness, Shannon's H and Peilou's J - across the different geographic groups in the Rampelli et al. 2017 study using the IV and GVD databases.

Supplementary Figure 4. The number of gut viral populations will still increase with more samples added to GVD. Collector's curve for gut viral populations in the GVD. (inset) Collector's curve for just the viromes from samples from the USA.

Supplementary Table 1. Origin of datasets and associated metadata used to create the gut virome database.

Supplementary Table 2. Gut Viral Database contigs family-level taxonomy and putative hosts.

Supplementary Table 3. Distributions of viral populations across GVD samples.

Supplementary Table 4. Core, common, low-overlap, and unique GVD viral populations

Supplementary Table 5. Human gut virome studies since the end of 2017 


\section{METHODS}

Experimental Model and Subject Details. Gut virome database (GVD) studies were selected by doing a thorough and manually curated search of the Web of Science Core Collection of Thomson Reuters for studies looking at viruses in the gut published prior to 2018. All studies that used next-generation sequencing and looked for viruses within the gut microbiome were selected to be part of GVD (see full list of studies in Supplementary Table 1). Additionally, we were given access to the reads of two studies that were unpublished at the time. One of the studies, however, is now published (Yinda et al., 2019).

Viral contig assembly, identification, and dereplication. Previously published GVD reads were downloaded from their respective hosting databases (e.g. SRA, iVirus, or MG-RAST). Prior work revealed that an individual's gut virome is stable across time (Minot et al., 2013), so reads were pooled per individual regardless of the number of time points, with a few exceptions (Fig. 1). These exceptions included studies with fecal microbiota transfers and studies whose reads were given to us prior to publication. For fecal microbiota transfers, all time points per individual were kept separate and processed independently. Read sets from two studies were given to us prior to publication (Yinda et al., 2019; Neto et al., unpublished). For the Yinda et al., 2019 study, individual's reads were pooled based on their location, age, and contact with bats. The pools were counted as a single individual's virome for our analyses. For the Nadia et al., (unpublished), all reads from all individuals were pooled together. A global map showing the number of individuals (or pooled read sets) originating from each country was created using the R packages 'rworldmap.' In total, there were 648 GVD samples from 572 individuals.

Pooled reads were then assembled using metaSPAdes 3.11.1 ${ }^{78}$. Following assembly, contigs $\geq 1.5 \mathrm{~kb}$ were piped through VirSorter ${ }^{79}$ and VirFinder ${ }^{80}$ and those that mapped to the human, cat or dog genomes were removed. For viral-enriched metagenomes (i.e. viromes), contigs $\geq 5 \mathrm{~kb}$ or $\geq 1.5 \mathrm{~kb}$ and circular that were sorted as VirSorter categories 1-6 and/or VirFinder score $\geq 0.7$ and $p<0.05$ were pulled for further investigation. Of these contigs, those sorted as VirSorter categories 1 and 2, VirFinder score $\geq 0.9$ and $p<0.05$ or were identified as viral by both VirSorter (categories 1-6) and VirFinder (score $\geq 0.7$ and $p<0.05$ ) were classified as viral. The remaining contigs were run through $\mathrm{CAT}^{81}$ and those with $<40 \%$ (based on an average gene size of 1000) of the genome classified as bacterial, archaeal, or eukaryotic were considered viral. For the microbial metagenomes, we took a more conservative approach with only contigs $\geq 5 \mathrm{~kb}$ or $\geq 1.5 \mathrm{~kb}$ and circular that were sorted as VirSorter categories $1-2$ and VirFinder score $\geq 0.6$ and $p<0.05$ were considered viral. Across the both the viral-enriched and microbial metagenomes, contigs $\geq 5 \mathrm{~kb}$ or $\geq 1.5 \mathrm{~kb}$ and circular that were classified as eukaryotic viral contigs by CAT were also considered viral. In total, 29,345 viral contigs were identified.

Viral contigs that were from known ssDNA or RNA viral families using CAT were grouped into populations if they shared $\geq 95 \%$ nucleotide identity across $\geq 100 \%$ of the genome. Because there are no benchmarked metagenomic population boundaries for ssDNA and RNA viral families, we chose to not use stringent dereplication. All other contigs were considered double-stranded DNA and were grouped into populations if they shared $\geq 95 \%$ nucleotide identity across $\geq 70 \%$ of the genome $\left(\right.$ sensu $\left.^{82}\right)$ using nucmer ${ }^{83}$. All the viral contigs that were assembled were dereplicated per study to create the individual virome (IV) databases and across all of GVD (see Supplementary Fig. 1). For GVD, this resulted in 434 13,203 total viral populations found in GVD (see Supplementary Table 3 for VirSorter, VirFinder, and CAT results), of which 6,373 were $\geq 10 \mathrm{~kb}$ in length. 
Core Viral Population Analyses. To explore if there were any core viral populations, the abundance table was turned into a binary presence-absence matrix. The number of GVD samples that each viral population was detected within was then calculated using $\mathrm{R}$ and divided by the total number (648) to get the percentage of samples. Each viral population's percentage was plotted in hive plot using 'geom_curve' in ggplot $2{ }^{84}$. This process was repeated on subsets of the matrix including all healthy individuals and only the healthy western adults. The number of viral populations that were present across different percentages were calculated using $\mathrm{R}$ and their distributions plotted using 'geom_histogram' in ggplot $2{ }^{84}$. CrAssphage viral populations in GVD were identified using CAT results and by dereplicating GVD viral populations with the crAssphage genomes identified in Guerin et al. ${ }^{64}$ and seeing which GVD genomes cluster. In total, there were 95 unique crAssphage populations. The binary presence-absence data for the crAssphage populations were plotted using pheatmap in $\mathrm{R}$.

Viral taxonomy. For each viral population, ORFs were called using Prodigal ${ }^{85}$ and the resulting protein sequences were used as input for vConTACT2 ${ }^{47}$ and for BLASTp. Double-stranded DNA viral populations represented by contigs $>10 \mathrm{~kb}$ were clustered with Viral RefSeq release 88 viral genomes using vConTACT2. Those that clustered with a virus from RefSeq based on amino acid homology based on DIAMOND ${ }^{86}$ alignments were able to be assigned to a known viral taxonomic genera. For viral dsDNA populations that could not be assigned taxonomy or were $<10 \mathrm{~kb}$, family level taxonomy was assigned using a majority-rules approach, where if $>50 \%$ of a genome's proteins were assigned to the same viral family using a blastp bitscore $\geq 50$ with a Viral RefSeq virus, it was considered part of that viral family (see Supplementary Table 3 for family-level taxonomy). For ssDNA and RNA viruses, CAT was used to assign the viral family (see Supplementary Table 3 for family-level taxonomy).

Viral Host Prediction. Bacteriophage hosts were predicted using a variety of bioinformatic methods including: (i) CRISPR-spacer matches, (ii) prophage blasts, (iii) tRNA genes matches, and (iv) WiSH matches ${ }^{87}$ against Bacterial Refseq v88. CRISPR spacers were predicted using MinCED (https://github.com/ctSkennerton/minced) and the CRISPR Recognition Tool (CRT ${ }^{88}$ ) and a BLASTn (task blastn-short -word_size 5) was used to assess matches between the CRISPR spacers and viral populations in GVD. Those with 1 mismatch were considered a match. For prophage blasts, a blastn of the viral population against Bacterial RefSeq was performed. A bacterial genome with $\geq 2500 \mathrm{bp}$ regions of their genome matching at 95\%ID with a viral population genome were considered putative hosts of that viral population $\left(\right.$ see $^{76}$ ). Viral tRNA genes and Bacterial RefSeq tRNA genes were predicted using tRNA-scan ${ }^{89}$ and then a blastn was performed between the viral and bacterial tRNA genes. Bacterial tRNA genes that matched viral tRNA genes at 95\% ID across $100 \%$ of the length were considered putative bacterial hosts. Lastly, WIsH was used to predict hosts according to default settings ${ }^{87}$. Priority host assignment was given to CRISPR, then prophage, WIsH and tRNA results. Viruses with putative archaeal hosts were predicted using MarVD ${ }^{90}$. Viruses with predicted eukaryotic hosts were assigned based on their assigned taxonomic viral family. the different viral populations in each sample, reads from each GVD pooled read set were first nondeterministically mapped to all GVD viral population genomes using bowtie2. Further, reads from each 
identity to the contigs, bedtools genomecov ${ }^{91}$ was used to determine how many positions across each genome were covered by reads, and custom Perl scripts were used to further filter out contigs without enough coverage across the length of the contig. All contigs $\leq 5 \mathrm{~kb}$ in length with $>70 \%$ of the contig covered were considered detected in the sample. Contigs $>5 \mathrm{~kb}$ in length with $\geq 5 \mathrm{~kb}$ in length covered were also considered detected in the sample ${ }^{92}$. BamM was used to calculate the average read depth ('tpmean' minus the top and bottom $10 \%$ depths) across each detected contig. For the alpha-diversity calculations, the average read depth was used as a proxy for abundance and normalized by total read number per metagenome to allow for sample-to-sample comparison. However, because most of the studies in GVD involved MDA, which can skew abundances, we chose to use only a presence-absence statistic (richness) for most of our $\alpha$-diversity calculations. Collector's curves and the whole GVD and across only American samples were calculated using the function 'specaccum' in the $\mathrm{R}$ 'vegan' package ${ }^{93}$.

Comparisons to IMG/VR, Viral RefSeq v88, and IV databases. The IMG/VR (1.1.2018 release) included all viral contigs assembled from different datasets. All of the viral contigs in GVD, Viral Refseq v88, and IV databases are dereplicated at the population level. In order to make IMG/VR comparable to GVD, Viral Refseq and IV databases, we needed to dereplicate the IMG/VR database. IMG/VR (1.1.2018 release) is composed of 715,672 contigs. Because dereplication is extremely computationally intensive, we decided to only focus on dereplicating viral contigs that originated from the human gut and had at least 1 read from a GVD metagenome map. These IMG/VR viral contigs were then dereplicated using the same methodology as previously described in the methods section. In total, 29,378 IMG/VR viral contigs were dereplicated into 6,652 viral populations. GVD pooled read sets were mapped to this IMG/VR human gut viral population database, Viral RefSeq v88, and the IV databases for each individual study in GVD. The raw abundances of the different IMG/VR and Viral RefSeq viral populations in each sample were calculated the same way as described in the previous section. The total number of viral populations detected per sample per study using the different databases were then plotted and comparative statistics using the 'ggboxplot' function from the 'ggpubr' package in R.

All of the viral populations from GVD, the dereplicated IMG/VR gut-specific dataset, and Viral Refseq were then dereplicated to see how many viral populations overlapped between databases. The results were then plotted using the 'VennDiagram' package in R. Importantly, in the dereplication process, some of the original viral populations in each database may be dereplicated down due to the presence of a longer viral contig from the same population that links the two together into the same population. Across the databases, 329, 177, and 459 viral populations were dereplicated in GVD, IMG/VR, and Viral Refseq, respectively. This is why the total number of populations displayed in the Venn diagram does not add up to the total number of viral populations in each database.

Clustering studies based on shared viral populations. To test how studies clustered together, the viral population presence-absence data from individuals (or pooled read sets) within a study were merged. In Study 1, individual A had viral population 1, 2, 4, 5 and individual B had viral population 3, then Study 1 had viral populations 1, 2, 3, 4, and 5. The different studies were then assessed for the number of shared viral populations that were present in both studies. These values were then displayed and hierarchically clustered using the $\mathrm{R}$ 'pheatmap' package and the stability of the hierarchical clusters were assess using the R 'pvclust' package. The number of shared viral populations between individuals (or pooled read sets within a sample) were clustered using the $\mathrm{R}$ 'SPIEC-EASI' package ${ }^{94}$ using the Meinshausen and 
524 Bühlmann (MB) method to infer associations between samples based on the shared number of viral populations. The network was plotted using the $\mathrm{R}$ ‘igraph’ package.

Alpha- and Beta-Diversity calculations. The $\alpha$ - (Richness, Shannon's $H$, and Peilous' $J$ ) and $\beta$ - (BrayCurtis dissimilarity) diversity statistics were performed using VEGAN ${ }^{93}$ in R. For all studies, except for Rampelli et al. ${ }^{26}$, only richness was calculated for both abundances based on read mapping to IMG/VR, Viral Refseq, the IV databases and GVD. Comparisons were plotted using 'ggboxplot' function in the R 'ggpubr' package. The Rampelli et al. ${ }^{26}$ did not use MDA, so we went ahead with scaling the raw abundances based on the number of quality controlled base pairs sequenced to normalize the data. All $\alpha$ diversity statistics were calculated and $\beta$-diversity was used to look at community structure using both the IV and GVD databases. Principal Coordinate analysis (function capscale of VEGAN package with no constraints applied) was used as the ordination method to plot the Bray-Curtis dissimilarity matrices (function vegdist; method "bray") after a cube root transformation (function nthroot; $n=3$ ). To determine if the Rampelli et al. samples clustered by geographic region, a permanova test (function "adonis") and the 95\% confidence interval were plotted using function "ordiellipse." Further, the samples were hierarchically clustered and plotted within the PCoA. To specifically look at abundance differences in the most abundant viral populations in the Rampelli et al. ${ }^{26}$ study, viral populations that were present in 50\% study individuals and their hosts information were plotted using the $\mathrm{R}$ 'pheatmap' package.

544 Gut_Virome_Database.

Data availability. All raw reads are available through SRA, iVirus, or MG-RAST using the identifiers

552 1. Clemente, J. C., Ursell, L. K., Parfrey, L. W. \& Knight, R. The impact of the gut microbiota on human health: An integrative view. Cell (2012). doi:10.1016/j.cell.2012.01.035

2. Lynch, S. V. \& Pedersen, O. The Human Intestinal Microbiome in Health and Disease. N. Engl. J. Med. 375, 2369-2379 (2016).

3. Gilbert, J. A. et al. Current understanding of the human microbiome. Nat. Med. (2018).

558 4. Schmidt, T. S. B., Raes, J. \& Bork, P. The Human Gut Microbiome: From Association to Modulation. Cell (2018). doi:10.1016/j.cell.2018.02.044

5. Frank, D. N. et al. Molecular-phylogenetic characterization of microbial community imbalances in 
human inflammatory bowel diseases. Proc. Natl. Acad. Sci. U. S. A. 104, 13780-5 (2007).

6. Huttenhower, C. \& Human Microbiome Project Consortium. Structure, function and diversity of the healthy human microbiome. Nature 486, 207-14 (2012).

564 7. Qin, J. et al. A metagenome-wide association study of gut microbiota in type 2 diabetes. Nature 490, 55-60 (2012).

8. Turnbaugh, P. J. et al. An obesity-associated gut microbiome with increased capacity for energy harvest. Nature 444, 1027-1031 (2006).

9. Yoshimoto, S. et al. Obesity-induced gut microbial metabolite promotes liver cancer through senescence secretome. Nature 499, 97-101 (2013).

10. Mirzaei, M. K. \& Maurice, C. F. Ménage à trois in the human gut: Interactions between host, bacteria and phages. Nature Reviews Microbiology 15, 397-408 (2017).

11. Schreiner, Andrew B., John Y. Kao, and V. B. Y. The gut microbiome in health and in disease. Curr. Opin. Gastroenterol. 31, 69-75 (2015).

12. Zhang, Y.-J. et al. Impacts of Gut Bacteria on Human Health and Diseases. Int. J. Mol. Sci. 16, 7493-7519 (2015).

13. Ogilvie, L. A. \& Jones, B. V. The human gut virome: A multifaceted majority. Frontiers in Microbiology 6, (2015).

14. Tetz, G. V. et al. Bacteriophages as potential new mammalian pathogens. Sci. Rep. 7, (2017).

15. Keen, E. C. \& Dantas, G. Close Encounters of Three Kinds: Bacteriophages, Commensal Bacteria, and Host Immunity. Trends Microbiol. 26, 943-954 (2018).

16. Rohwer, F. \& Edwards, R. The phage proteomic tree: A genome-based taxonomy for phage. $J$.

583 17. Roux, S. et al. Minimum Information about an Uncultivated Virus Genome (MIUViG): a

18. Paez-Espino, D. et al. IMG/VR: a database of cultured and uncultured DNA Viruses and retroviruses. Nucleic Acids Res. 45, D457-D465 (2017).

19. Lim, E. S. et al. Early life dynamics of the human gut virome and bacterial microbiome in infants.

590 20. Reyes, A. et al. Viruses in the faecal microbiota of monozygotic twins and their mothers. Nature

$591 \quad$ 466, 334-338 (2010).

592 21. Ly, M. et al. Transmission of viruses via our microbiomes. Microbiome 4, 64 (2016).

593 22. Manrique, P. et al. Healthy human gut phageome. Proc. Natl. Acad. Sci. 113, 10400-10405

$594 \quad$ (2016). 
23. Minot, S. et al. The human gut virome: Inter-individual variation and dynamic response to diet. Genome Res. 21, 1616-1625 (2011).

24. Minot, S., Grunberg, S., Wu, G. D., Lewis, J. D. \& Bushman, F. D. Hypervariable loci in the human gut virome. Proc. Natl. Acad. Sci. 109, 3962-3966 (2012).

25. Minot, S. et al. Rapid evolution of the human gut virome. Proc. Natl. Acad. Sci. 110, 12450 12455 (2013).

601 26. Rampelli, S. et al. Characterization of the human DNA gut virome across populations with different subsistence strategies and geographical origin. Environ. Microbiol. 19, 4728-4735 (2017).

27. Broecker, F., Klumpp, J. \& Moelling, K. Long-term microbiota and virome in a Zürich patient after fecal transplantation against Clostridium difficile infection. Ann. N. Y. Acad. Sci. 1372, 29-

30. Kang, D. W. et al. Microbiota Transfer Therapy alters gut ecosystem and improves gastrointestinal 41 (2016).

31. Zuo, T. et al. Bacteriophage transfer during faecal microbiota transplantation in Clostridium difficile infection is associated with treatment outcome. Gut 67, 634-643 (2017).

32. Norman, J. M. et al. Disease-specific alterations in the enteric virome in inflammatory bowel disease. Cell 160, 447-460 (2015).

33. Pérez-Brocal, V. et al. Study of the viral and microbial communities associated with Crohn's disease: A metagenomic approach. Clin. Transl. Gastroenterol. 4, (2013).

34. Monaco, C. L. et al. Altered Virome and Bacterial Microbiome in Human Immunodeficiency Virus-Associated Acquired Immunodeficiency Syndrome. Cell Host Microbe 19, 311-322 (2016).

35. Kramná, L. et al. Gut virome sequencing in children with early islet autoimmunity. Diabetes Care 38, 930-933 (2015).

36. Zhao, G. et al. Intestinal virome changes precede autoimmunity in type I diabetes-susceptible children. Proc. Natl. Acad. Sci. 201706359 (2017). doi:10.1073/pnas.1706359114 encephalomyelitis/chronic fatigue syndrome differ in physiological parameters and gut 
microbiome composition. Am. J. Case Rep. 17, 720-729 (2016).

39. Gregory, A. C. et al. Genomic differentiation among wild cyanophages despite widespread horizontal gene transfer. BMC Genomics 17, (2016).

632 40. Brum, J. R. et al. Ocean Viral Communities. Science (80-. ). 348, 1261498-1-11 (2015).

633 41. Gregory, A. et al. Marine viral macro- and micro-diversity from pole to pole. Cell (2019).

634 42. Duhaime, M. B. \& Sullivan, M. B. Ocean viruses: Rigorously evaluating the metagenomic 635 sample-to-sequence pipeline. Virology 434, 181-186 (2012).

636 43. Duhaime, M. B. et al. Comparative omics and trait analyses of marine Pseudoalteromonas phages advance the phage OTU concept. Front. Microbiol. (2017). doi:10.3389/fmicb.2017.01241

45. Jang, H. Bin et al. Gene sharing networks to automate genome-based prokaryotic viral taxonomy. bioRxiv (2019). doi:https://doi.org/10.1101/533240

46. Bolduc, B. et al. vConTACT: an iVirus tool to classify double-stranded DNA viruses that infect Archaea and Bacteria. PeerJ 5, e3243 (2017).

47. Bin Jang, H. et al. Taxonomic assignment of uncultivated prokaryotic virus genomes is enabled by gene-sharing networks. Nat. Biotechnol. (2019). doi:10.1038/s41587-019-0100-8

48. Monaco, C. \& Kwon, D. Next-generation Sequencing of the DNA Virome from Fecal Samples. BIO-PROTOCOL (2017). doi:10.21769/bioprotoc.2159

49. Eckburg, P. B. et al. Microbiology: Diversity of the human intestinal microbial flora. Science (80-. ). (2005). doi:10.1126/science.1110591

50. Ott, S. J. et al. Reduction in diversity of the colonic mucosa associated bacterial microflora in patients with active inflammatory bowel disease. Gut (2004). doi:10.1136/gut.2003.025403

51. Ley, R. E. et al. Obesity alters gut microbial ecology. Proc. Natl. Acad. Sci. U. S. A. (2005). doi:10.1073/pnas.0504978102

52. Nicholson, J. K. et al. Host-gut microbiota metabolic interactions. Science (2012). doi:10.1126/science.1223813

53. Paez-Espino, D. et al. IMG/VR v.2.0: an integrated data management and analysis system for cultivated and environmental viral genomes. Nucleic Acids Res. gky1127-gky1127 (2018).

658 55. Zhang, K. et al. Sequencing genomes from single cells by polymerase cloning. Nat. Biotechnol. 659 (2006). doi:10.1038/nbt1214

660 56. Woyke, T. et al. Assembling the marine metagenome, one cell at a time. PLoS One (2009). 661 doi:10.1371/journal.pone.0005299

662 57. Yilmaz, S., Allgaier, M. \& Hugenholtz, P. Multiple displacement amplification compromises 
quantitative analysis of metagenomes. Nat. Methods 7, 943-944 (2010).

664 58. Kim, K.-H. \& Bae, J.-W. Amplification methods bias metagenomic libraries of uncultured singlestranded and double-stranded DNA viruses. Appl. Environ. Microbiol. 77, 7663-8 (2011).

59. Marine, R. et al. Caught in the middle with multiple displacement amplification: The myth of pooling for avoiding multiple displacement amplification bias in a metagenome. Microbiome 2, 18 (2014).

60. Kim, K. H. et al. Amplification of uncultured single-stranded DNA viruses from rice paddy soil. Appl. Environ. Microbiol. 74, 5975-5985 (2008).

61. Roux, S. et al. Towards quantitative viromics for both double-stranded and single-stranded DNA viruses. PeerJ 4, e2777 (2016).

63. Mayo-Muñoz, D. Viral genome isolation from human faeces for succession assessment of the human gut virome. in Methods in Molecular Biology (2018). doi:10.1007/978-1-4939-8682-

64. Guerin, E. et al. Biology and Taxonomy of crAss-like Bacteriophages, the Most Abundant Virus

65. Franzosa, E. A. et al. Identifying personal microbiomes using metagenomic codes. Proc. Natl.

66. Norman, J. M. et al. Disease-specific alterations in the enteric virome in inflammatory bowel disease. Cell 160, 447-460 (2015).

67. Lemos, L. N., Fulthorpe, R. R., Triplett, E. W. \& Roesch, L. F. W. Rethinking microbial diversity analysis in the high throughput sequencing era. J. Microbiol. Methods (2011). doi:10.1016/j.mimet.2011.03.014

68. Yatsunenko, T. et al. Human gut microbiome viewed across age and geography. Nature (2012). doi:10.1038/nature11053

69. De Filippo, C. et al. Impact of diet in shaping gut microbiota revealed by a comparative study in children from Europe and rural Africa. Proc. Natl. Acad. Sci. U. S. A. (2010).

693 70. Schnorr, S. L. et al. Gut microbiome of the Hadza hunter-gatherers. Nat. Commun. (2014).

694 doi:10.1038/ncomms4654

695 71. Yatsunenko, T. et al. Human gut microbiome viewed across age and geography. Nature 486, 222$696227(2012)$. 
72. Shkoporov, A. N. \& Hill, C. Bacteriophages of the Human Gut: The "Known Unknown" of the Microbiome. Cell Host Microbe 25, 195-209 (2019).

73. Emerson, J. B. et al. Host-linked soil viral ecology along a permafrost thaw gradient. Nat. Microbiol. (2018). doi:10.1038/s41564-018-0190-y

74. Paez-Espino, D. et al. Uncovering Earth's virome. Nature 536, 425-430 (2016).

75. Solonenko, S. a et al. Sequencing platform and library preparation choices impact viral metagenomes. BMC Genomics 14, 320 (2013).

76. Roux, S. et al. Ecogenomics and potential biogeochemical impacts of globally abundant ocean viruses. Nature 537, 689-693 (2016).

77. Pasolli, E. et al. Extensive Unexplored Human Microbiome Diversity Revealed by Over 150,000 Genomes from Metagenomes Spanning Age, Geography, and Lifestyle. Cell (2019).

80. Ren, J., Ahlgren, N. A., Lu, Y. Y., Fuhrman, J. A. \& Sun, F. VirFinder: a novel k-mer based tool doi:10.1016/j.cell.2019.01.001

81. Cambuy, D. D., Coutinho, F. H. \& Dutilh, B. E. Contig annotation tool CAT robustly classifies

82. Brum, J. R. et al. Patterns and ecological drivers of ocean viral communities. Science (80-. ). 348, (2015).

83. Kurtz, S. et al. Versatile and open software for comparing large genomes. Genome Biol. (2004). doi:10.1186/gb-2004-5-2-r12

84. Ginestet, C. ggplot2: Elegant Graphics for Data Analysis. J. R. Stat. Soc. Ser. A (Statistics Soc. (2011). doi:10.1111/j.1467-985x.2010.00676_9.x

85. Hyatt, D. et al. Prodigal: prokaryotic gene recognition and translation initiation site identification. BMC Bioinformatics 11, 119 (2010).

86. Buchfink, B., Xie, C. \& Huson, D. H. Fast and sensitive protein alignment using DIAMOND. Nat. Methods 12, 59-60 (2015).

727 87. Galiez, C., Siebert, M., Enault, F., Vincent, J. \& Söding, J. WIsH: who is the host? Predicting prokaryotic hosts from metagenomic phage contigs. Bioinformatics 33, 3113-3114 (2017).

88. Bland, C. et al. CRISPR recognition tool (CRT): a tool for automatic detection of clustered regularly interspaced palindromic repeats. BMC Bioinformatics 8, 209 (2007). 
731 89. Lowe, T. M. \& Eddy, S. R. tRNAscan-SE: A program for improved detection of transfer RNA genes in genomic sequence. Nucleic Acids Res. 25, 955-964 (1997).

733 90. Vik, D. R. et al. Putative archaeal viruses from the mesopelagic ocean. PeerJ 5, e3428 (2017).

734 91. Quinlan, A. R. \& Hall, I. M. BEDTools: A flexible suite of utilities for comparing genomic features. Bioinformatics (2010). doi:10.1093/bioinformatics/btq033

736 92. Ann Gregory, A. C. et al. Marine DNA Viral Macro-and Microdiversity from Pole to Pole. Cell 737 (2019). doi:10.1016/j.cell.2019.03.040

738 93. Dixon, P. VEGAN, a package of R functions for community ecology. Journal of Vegetation Science (2003). doi:10.1111/j.1654-1103.2003.tb02228.x

740 94. Kurtz, Z. D. et al. Sparse and Compositionally Robust Inference of Microbial Ecological Networks. PLoS Comput. Biol. (2015). doi:10.1371/journal.pcbi.1004226

ACKNOWLEDGEMENTS. Computational support was provided by an award from the Ohio

744 Supercomputer Center (OSC) to MBS. Study design and manuscript comments from Shini Sunagawa,

745 Miguelangel Cuenca Vera, Bas E. Dutilh, Ksenia Arkhipova, Pedro Meirelles and Simon Roux are

746 gratefully acknowledged. Funding was provided by the Gordon and Betty Moore Foundation (\#3790) to

747 MBS and an NIH T32 training grant fellowship (AI112542) to ACG.

AUTHOR CONTRIBUTIONS. A.C.G. collected all datasets and metadata for the study. A.C.G. and the data, and wrote the manuscript. All authors approved the final manuscript.

COMPETING INTERESTS. The authors declare no competing interests. 
A

Gut Viral Database

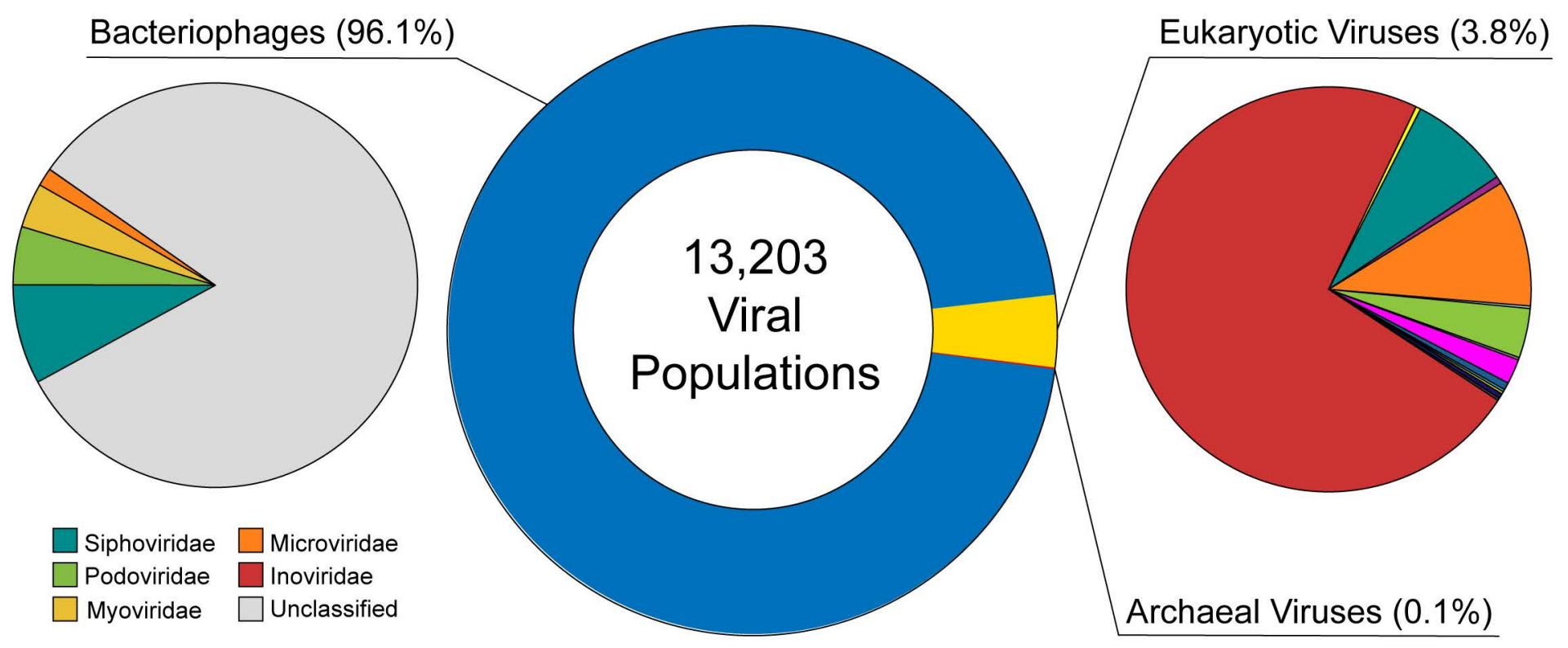

Adenoviridae

Alphaflexiviridae

$\square$ Anelloviridae

$\square$ Astroviridae

$\square$ Circoviridae

$\square$ Geminiviridae

$\square$ Genomoviridae

$\square$ Iflaviridae

$\square$ Papillomaviridae

$\square$ Parvoviridae

$\square$ Picornaviridae

$\square$ Polyomaviridae

$\square$ Rudiviridae

$\square$ Virgaviridae

Archaeal Viruses (0.1\%)

B

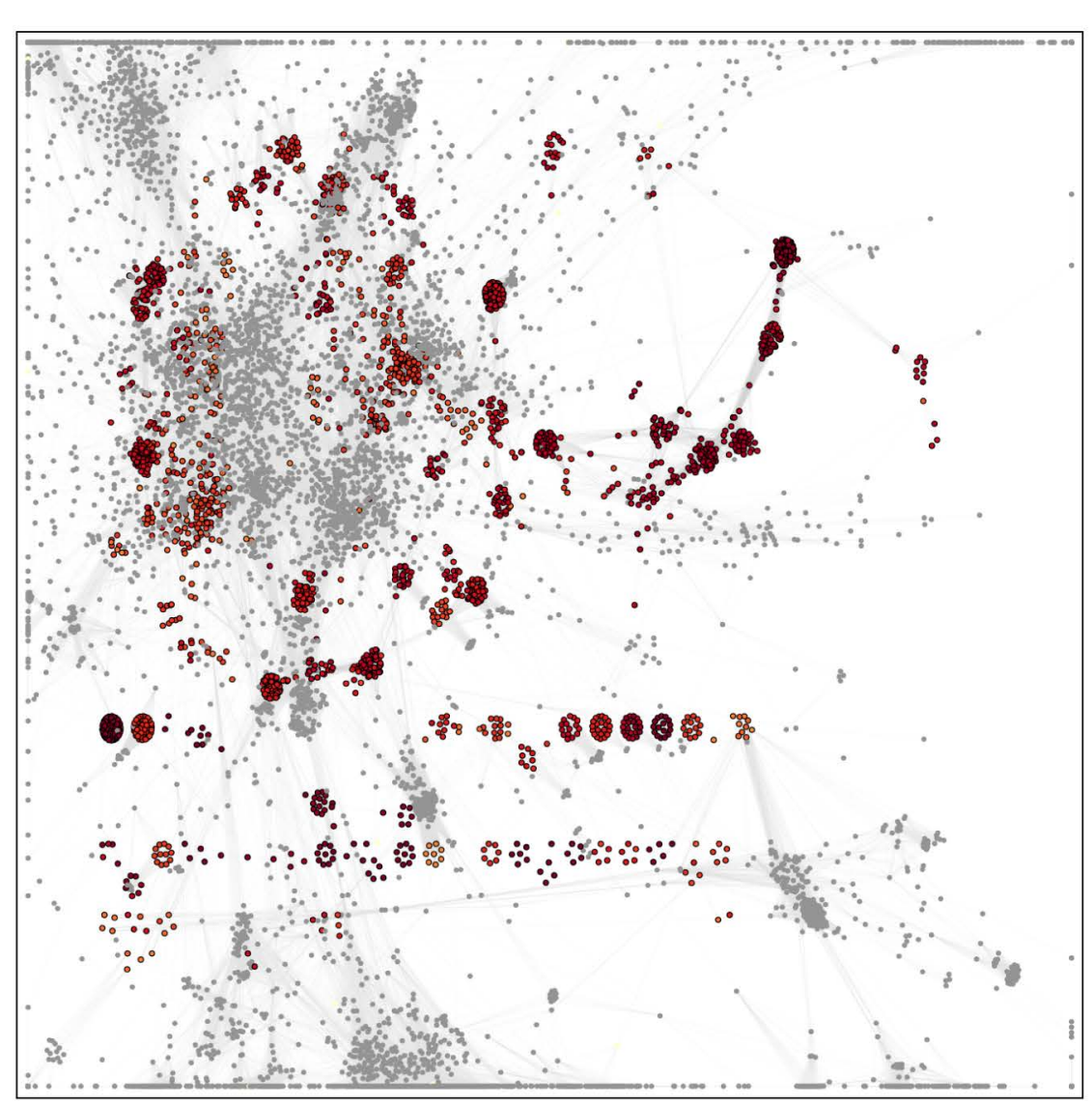

C

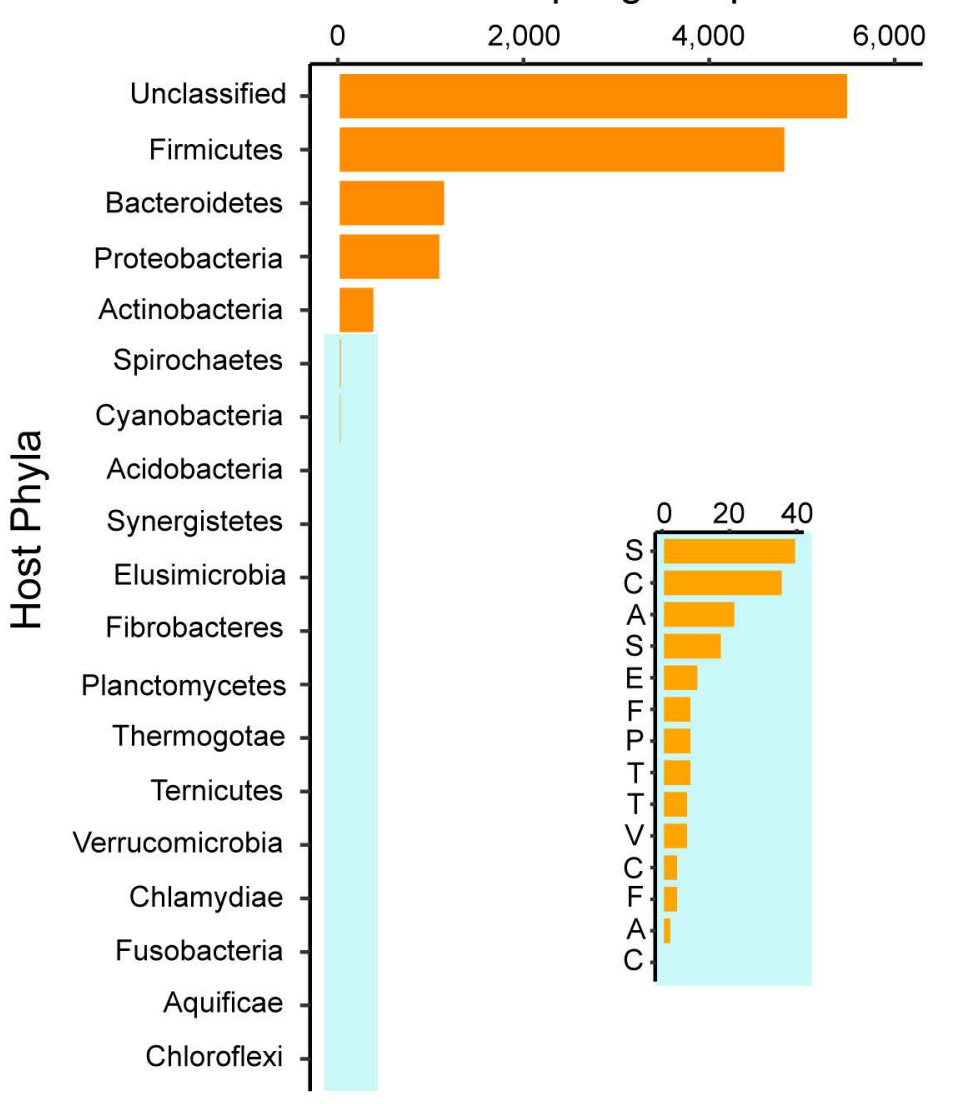




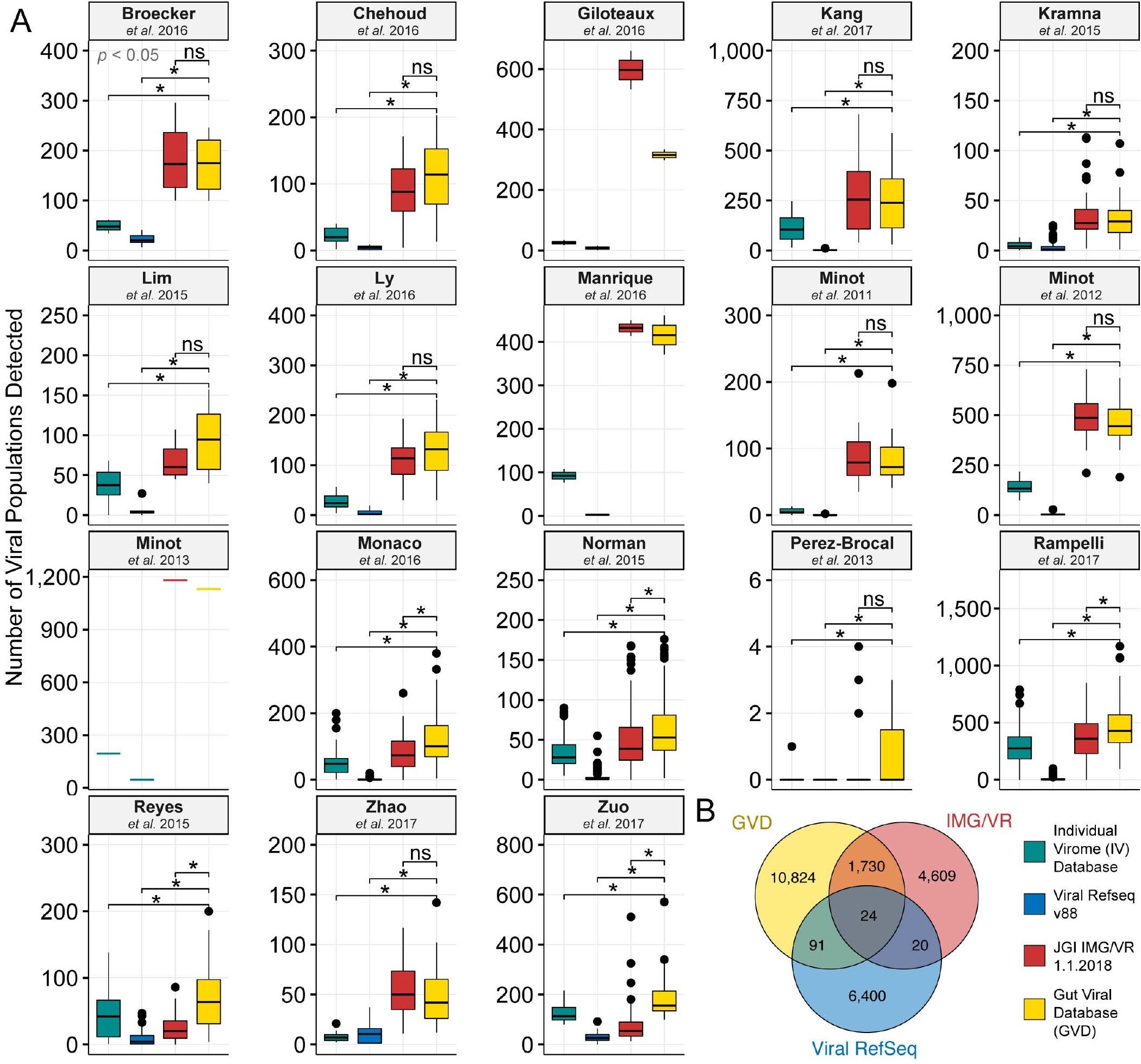




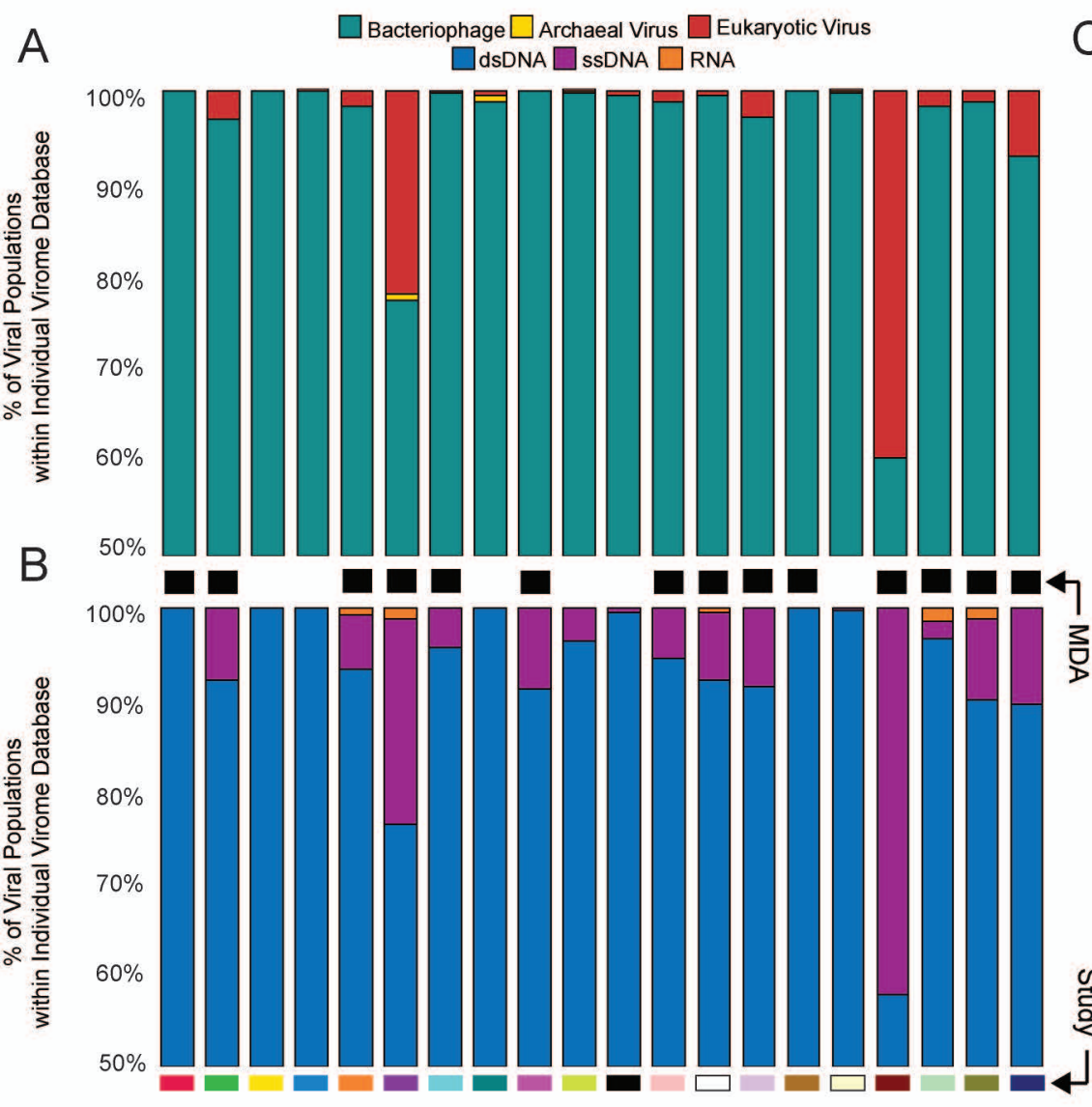

C

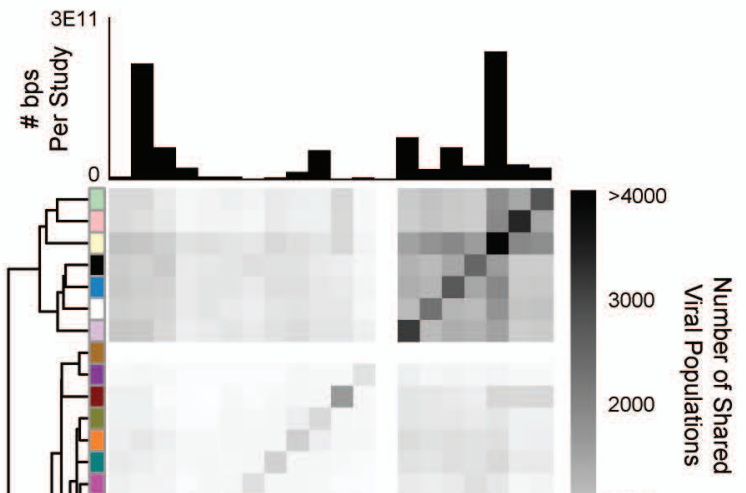

D
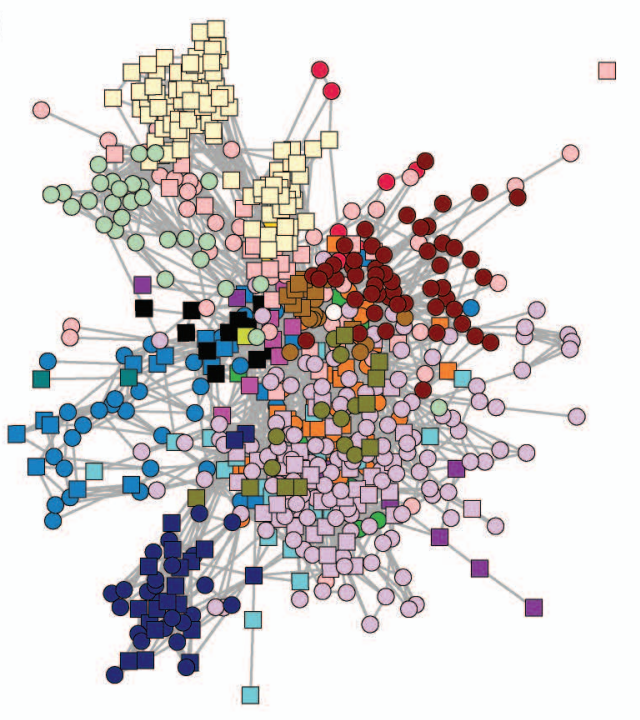

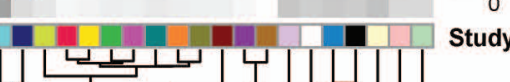

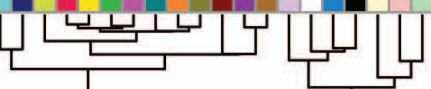

$\square$ Healthy

Other

\begin{tabular}{|r|lr|l|l|}
\hline Broecker et al. 2016 & Lim et al. 2015 & Minot et al. 2013 & Rampelli et al. 2017 \\
Chehoud et al. 2016 & Ly et al. 2016 & Monaco et al. 2016 & Reyes et al. 2015 \\
Giloteaux et al. 2016 & Manrique et al. 2016 & Neto (unpublished) & Yinda et al. 2019 (partia) \\
Kang et al. 2017 & Minot et al. 2011 r Norman et al. 2015 & Zhao et al. 2017 \\
Kramna et al. 2015 & Minot et al. 2012 Perez-Brocal et al. 2013 & Zuo et al. 2017 \\
\hline
\end{tabular}




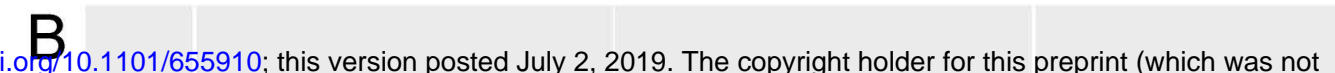

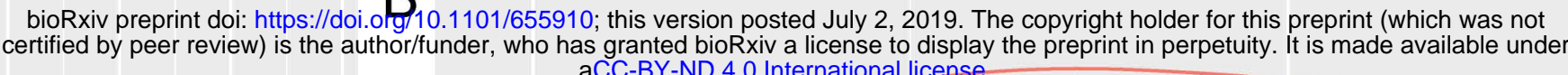

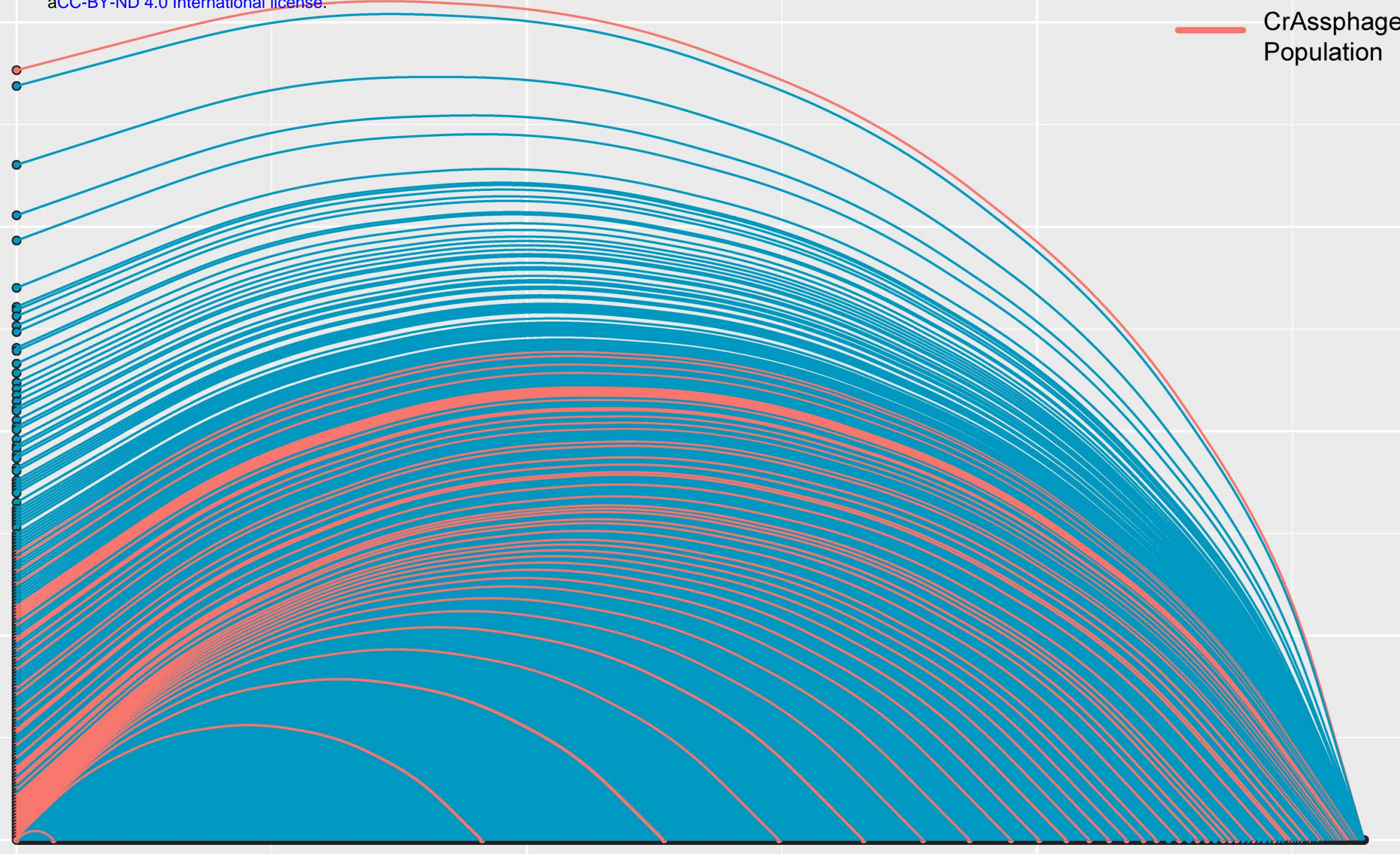

13,203 GVD Viral Populations

C
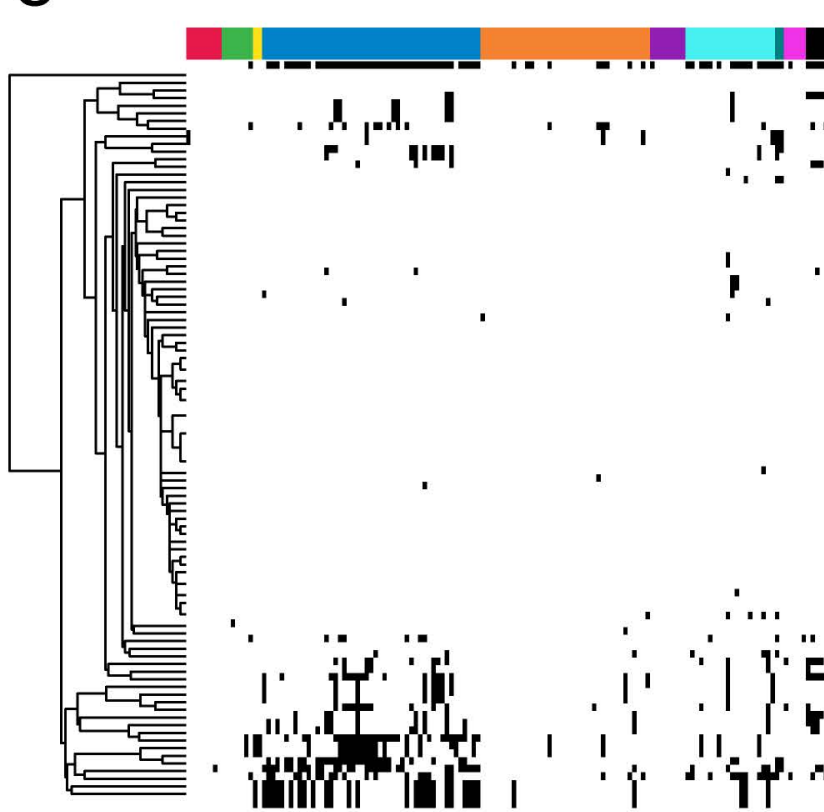

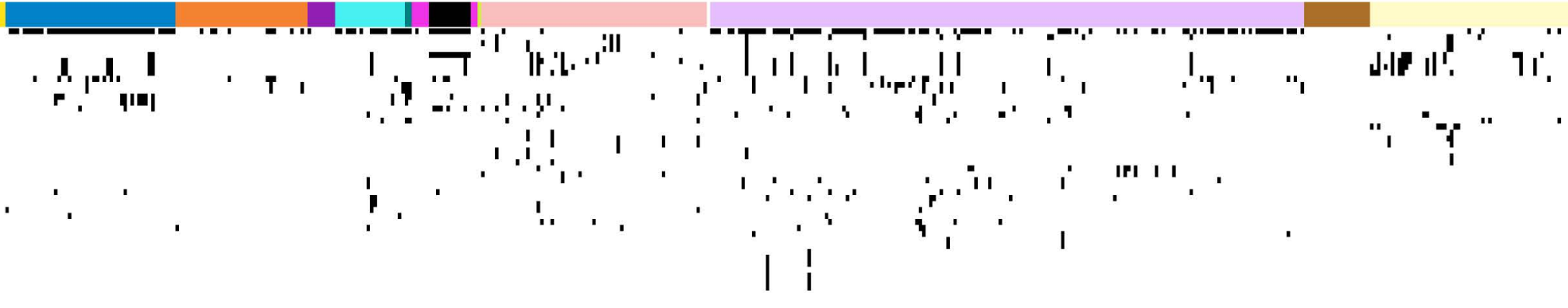

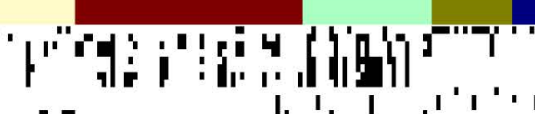

, 1 19

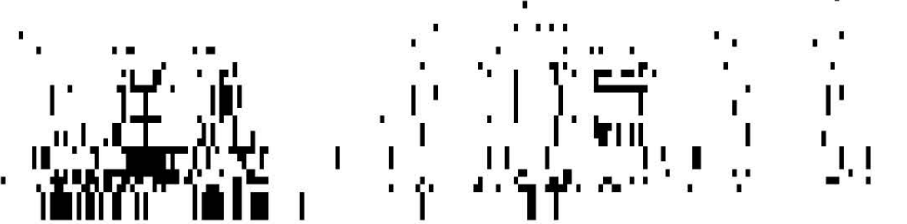

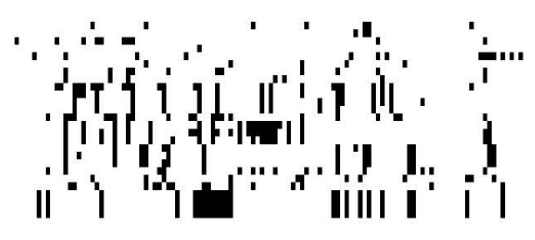

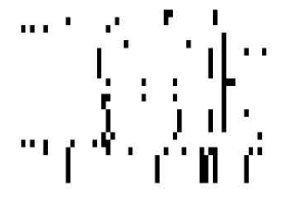

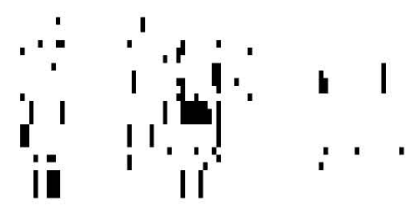

(1)

Broecker et al. 2016 Chehoud et al. 2016 Giloteaux et al. 2016 Kang et al. 2017 Kramna et al. 2015 Lim et al. 2015 Ly et al. 2016

Manrique et al. $2016 \square$ Minot et al. $2011 \square$ Minot et al. $2012 \square$ Minot et al. 2013 Monaco et al. $2016 \square$ Neto et al. (unpublished) Norman et al. 2015

Perez-Brocal et al. 2013 Rampelli et al. 2017 Reyes et al. 2015 Yinda et al. 2019 (partial) Zhao et al. 2017 Zuo et al. 2017 

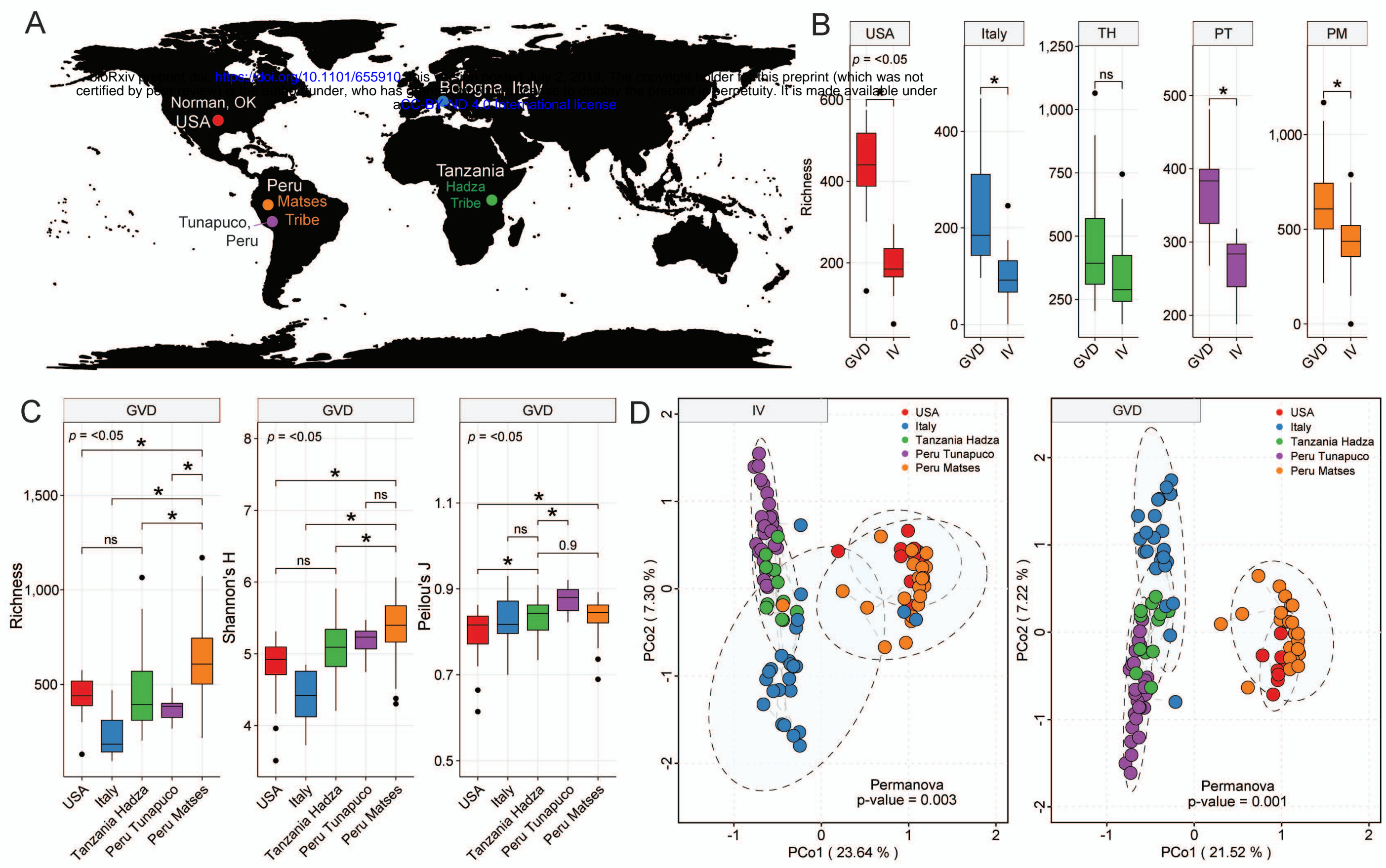

E

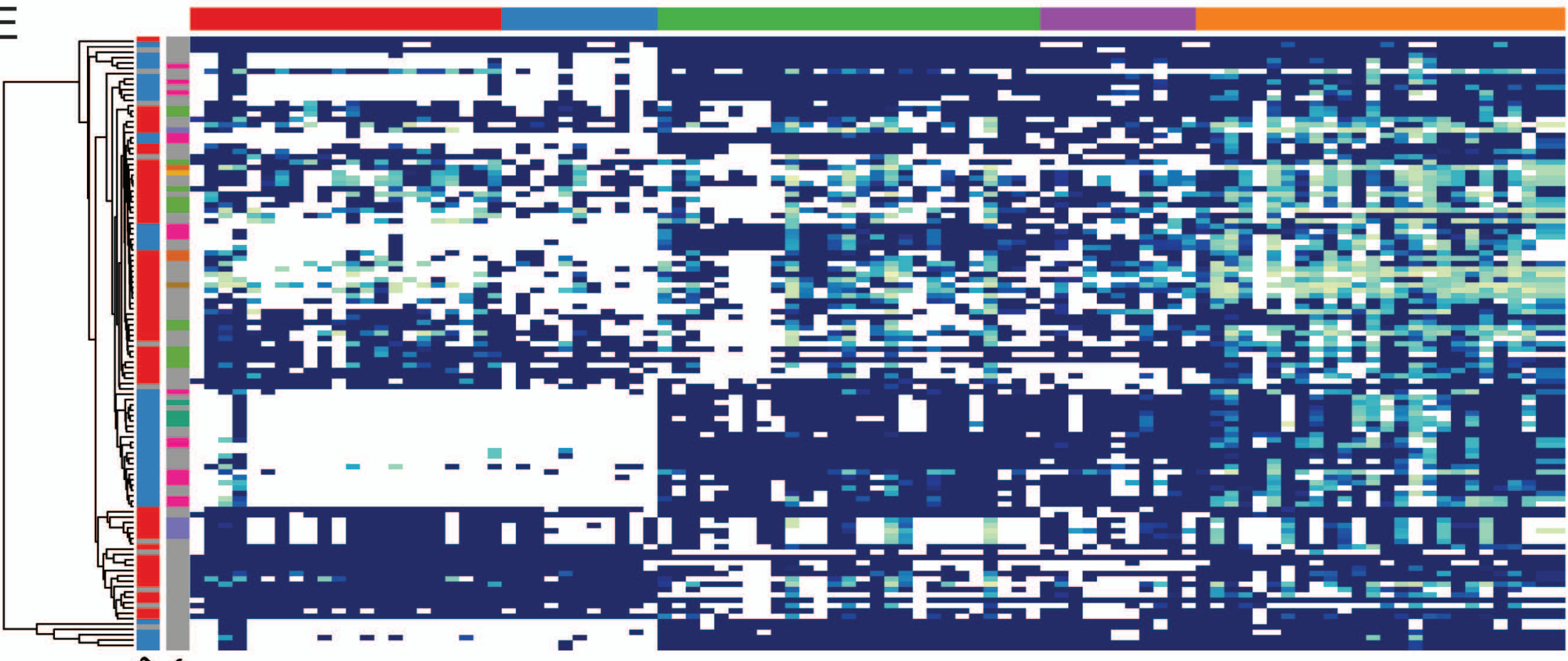

Region so 